\title{
Development of Materials Informatics Platform
}

\author{
Yasumitsu Orii $^{1^{*}}$, Shuichi Hirose ${ }^{2}$, Akihiro Fujita ${ }^{3}$, and Masakazu Kobayashi ${ }^{1}$ \\ ${ }^{1}$ New Value Creation Office, NAGASE \& Co.,LTD, \\ 5-1 Nihonbashi-Kobunacho, Chuo-ku, Tokyo,103-8355, Japan \\ ${ }^{2}$ NAGASE R\&D Center, New Value Creation Office, Nagase \& Co., LTD \\ 2-2-3, Murotani, Nishi-ku, Kobe city, Hyogo, 651-2241, Japan \\ ${ }^{3} R \& D$ Center, HAYASHIBARA \&CO., LTD. \\ 675-1, Fujisaki, Naka-ku, Okayama, 702-8006, Japan \\ *yasumitsu.orii@nagase.co.jp
}

\begin{abstract}
As the use of IT increases importance with big data and AI, the issue of power consumption has been highlighted. Under these circumstances, the development of new materials is more and more important. Materials Informatics (MI) is one of the hottest technologies in the material development field, because of its potential to reduce the time and costs of discovering innovative materials. To achieve this, the key is to collect data that has been accumulated for many years at research institutions and companies, and to make information extracted from the data into knowledge. This article introduces the development of two methods based on AI: the "cognitive approach", which reads vast amounts of literature information and digitizes data, and the "analytic approach", which theoretically estimates the structure and physical properties of chemical substances from predictive models.
\end{abstract}

Keywords: Materials Informatics, Cognitive approach, Analytics approach

\section{Introduction}

In terms of "data volume", the total amount of digital information generated worldwide in 2010 exceeded 1 ZB (Zettabyte) per year and reached at $44 \mathrm{ZB}$ in 2020[1]. The Internet of Things (IoT) is key factor of the data volume increasing. Conventionally, humans created data, but these days, machines and sensors create data on behalf of humans. And then, the data volume will expand to $163 \mathrm{ZB}$ in 2025[2].

However, among this immense amount of data, only about $20 \%$ of the data used by companies is structured data, and it is easy to manage with conventional computers. On the other hand, unstructured data accounts for about $80 \%$ of the total data, and it is expected that this percentage will increase furthermore soon. And that huge amounts of data will not be used. It is no exaggeration to say that AI has emerged to take full advantage of this buried unstructured data.

Regarding the "network", an increase in communication speed is a major interest. The data created by sensors is stored in various places, but most of them are stored in the cloud environment via wireless communication. At present, the communication band and speed are problems, and there are cases and situations where it is impossible to use, even if it is desired by users. The fifthgeneration mobile communication system (5G) is expected as a breakthrough. It is assumed that a maximum transmission speed of $20 \mathrm{Gbps}$, that is 20 times faster than current LTE, and low latency. Therefore, remote robot operation and surgery in real time can be performed. In addition, the problem of multiple simultaneous connections will be solved. $5 \mathrm{G}$ will be the key technology for acceleration of big data.

As for "hardware", along with Moore's Law, semiconductors have evolv4ed, that is, the performance of computers is one trillion times higher in 60 years and has evolved overwhelmingly. This is exactly following "The Law of Accelerating Returns"[3] proposed by Ray Kurzweil (Fig.1.). One important invention is linked to another, it can be shortening the time between the appearance of the next important invention and accelerating the 
speed of innovation. Thus, the performance of computers has been improved based on the rule of thumb that science and technology advance exponentially instead of linearly. Moore's law in the law of accelerating returns is said to be the fifth paradigm shift. The history of semiconductor starts from punch cards, relays, vacuum tubes, transistors, and integrated circuits. It is exactly that integrated circuits have evolved along with Moore's law. However, following Moore's law has also become more difficult due to the physical limitations of transistor integration. That means the improvement of AI performance requires the sixth paradigm shift. In order to bring about the sixth paradigm in the law of accelerating returns, the discovery of new materials is an important factor, and Materials Informatics (MI) [4],[5] is the powerful method to make it happen.

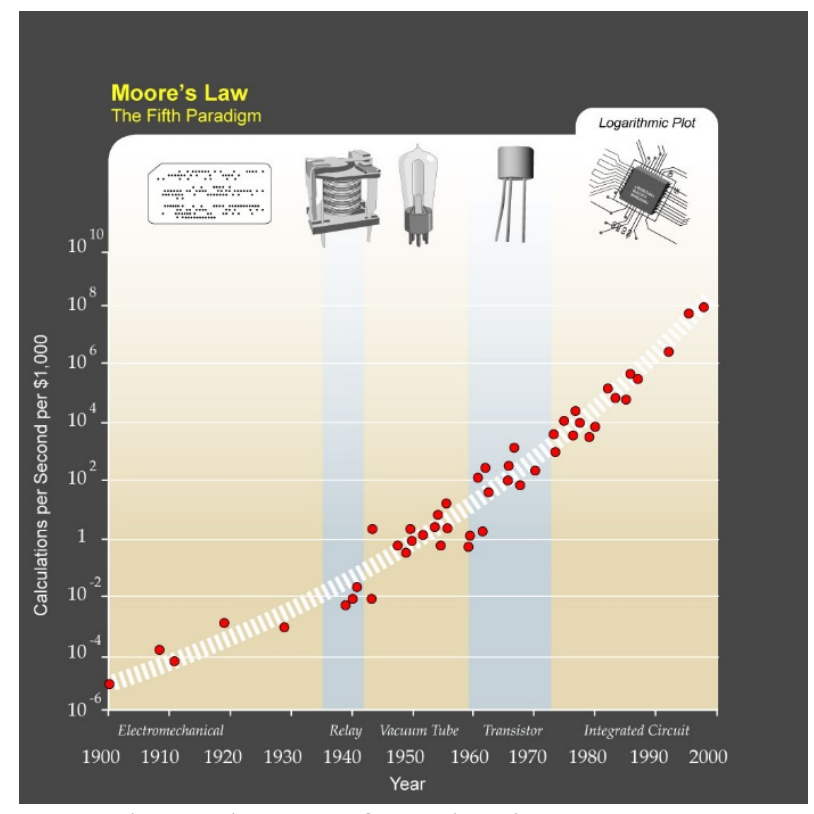

Fig. 1. The Law of Accelerating Returns.

\section{Cognitive approach and analytics approach}

According to Nature report about Chemical Space, there are $10^{9}$ (one billion) materials known to the world. On the other hand, there are $10^{62}$ unknown materials [6]. AI can find suitable materials from such a huge unknown materials. To achieve it, we developed the "cognitive approach" [7] and "analytic approach" [8] and their features are summarized in Fig. 2.

\begin{tabular}{|c|c|c|}
\hline $\begin{array}{l}\text { - Public documents } \\
\text { - In-house documents }\end{array}$ & Input data & $\begin{array}{r}\text { - Chemical structures and } \\
\text { properties }\end{array}$ \\
\hline $\begin{array}{l}\text { - NLP } \\
\text { - Data structuring rule }\end{array}$ & Theory & $\begin{array}{r}\text {-Statistical processing } \\
\cdot \text { Machine Learning }\end{array}$ \\
\hline - Novel knowledge & Output data & - Chemical structure \\
\hline
\end{tabular}

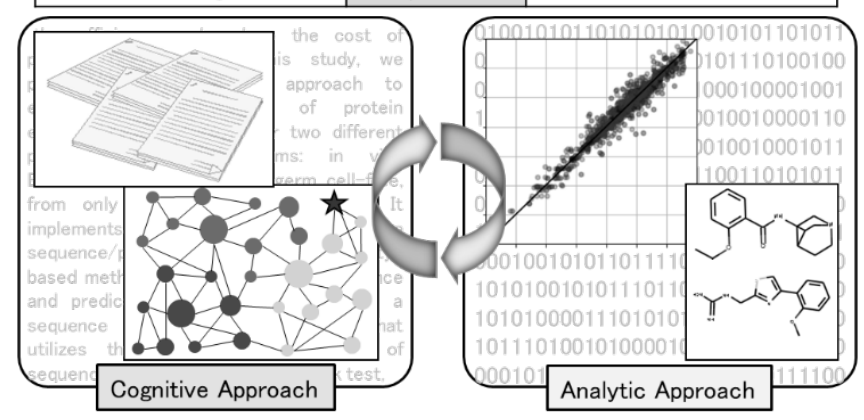

Fig. 2. Overview of cognitive and analytic approach.

The cognitive approach consists of three steps: "converting document information into structural data", "extracting facts by natural language processing", and "transforming facts into a knowledge graph (KG) expressing their correlation" (Fig. 3).

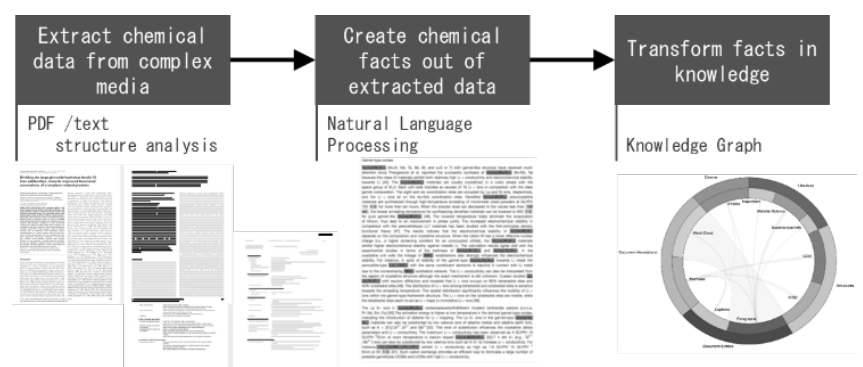

Fig. 3. Workflow for cognitive approach.

As a first step, the document information, which is unstructured data, is converted into structured data so that the computer can process it. For example, scientific papers, patents, and in-house documents are usually stored in pdf format, therefore those data are often difficult for computer to process. One of the solutions for document processing is the Corpus Conversion Service (CCS) reported by Staar et al. [9]. CCS can recognize titles and authors (labels) in scientific papers and extract text information along with the labels. In the next step, facts (entities) related to materials and their relationships (relation) are extracted from the text. Here, in addition to commonly used natural language processing, a dictionary that describes technical terms such as material names and physical property values and the relevance of those terms in the field of material is required to get entity. The final step is to develop the KG by joining the relations extracted from the document. The KG can 
represent data obtained from various resources as one graph structure.

While the cognitive approach has the advantage of handling volumes that humans can hardly read, it can only extract facts in documents. In the future, it is expected that cognitive approach will infer new knowledge not written in documents by combining $\mathrm{KG}$ and machine learning. That is, we call Inference Model (IM).

The analytic approach includes two processes. The one is called a forward problem that analyzes a correlation between chemical structures and their physical property values, and the other is called an inverse problem that generates a chemical structural formula satisfying a target property value (Fig. 4).

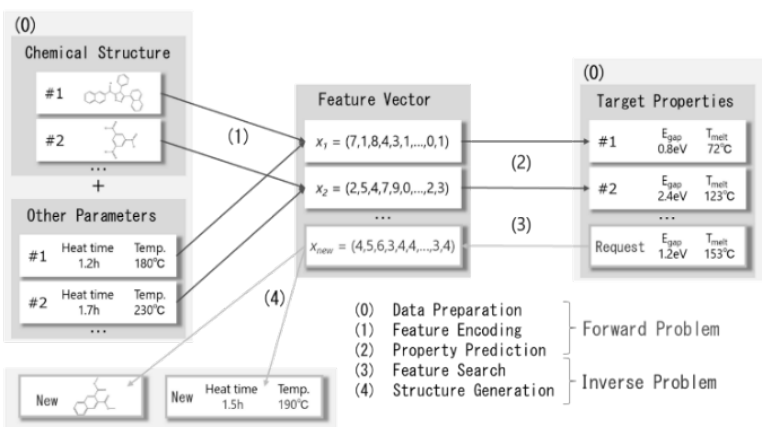

Fig. 4. Workflow for analytic approach.

First, the chemical structural formulas read as input information are converted into feature vectors using by a set of descriptors. For instance, the number of atoms, the number of partial structures, and so on are employed for descriptors. They need to be able to capture features of molecular structure exactly, and to generate a chemical structural formula from the features.

Next, model for predicting a physical property value is constructed. Generally, regression model is applied for such cases. The model with high prediction accuracy can be constructed, then it is possible to proceed to the next process called the inverse problem. In this step, a feature vector that satisfies a target property value is explored from a prediction model using a particle swarm optimization (PSO) method or other similar methods at the beginning.

Next, based on the given feature vector, chemical structural formulas that can exist mathematically are generated employing graph theory, and so on. As an application example of this method, Takeda et al. reported the design of drug candidate compounds [10]. In this research, candidate compounds which meet the target values for two contradictory property values (LogP(Octanol/water partition coefficient), TPSA(polar envelope)) are designed, some of them were not included in the input data set. The potential of analytic approach to discover novel materials is of great interest.

However, it is difficult for the inverse problem to design material with large molecular weight due to their limitation of the calculation cost of search space. In this case, designing sub-structure having a high correlation with the target property value is one of the solutions.

While analytic and cognitive approaches seem at first glance to be entirely different approaches, combining the two approaches is expected to further accelerate material discovery.

\section{KG construction}

\subsection{Biochemical KG}

In recent years, biochemistry, which mainly focuses on cells, microorganisms, viruses and biomolecules such as proteins, sugars, lipids, DNA and RNA, has become increasingly important in contributing to human health and solving medical problems. In the field of biochemistry, it is widely practiced to register research data in public databases on the Internet after publishing papers and patents. Each of these public databases has its purposes, and the data which meet those purposes are registered in the databases. For example, Uniprot[11] and Genbank[12] are comprehensive databases on proteins and genes. CAZy[13] is a database which specializes in enzymes related to the degradation and production of carbohydrates. BRENDA[14] is a comprehensive database specialized for enzymatic reaction. Searching in single database will not yield sufficient results because each database has only the data that meet the purposes. Therefore, biochemists need to search multiple databases to obtain the data they need. Furthermore, some data are only described in papers and not registered in databases, making it difficult to obtain the necessary information. To address these issues, it is necessary to construct a queryable knowledge graph where research data retrieved from multiple databases and a large number of papers are systematically organized. NAGASE CO., LTD., HAYASHIBARA CO., LTD and IBM Zurich constructed the BioChemical Knowledge Graph (BCKG) from seven databases, papers and books . Furthermore, in this paper, we demonstrated an efficient and comprehensive search for carbohydrate-active enzymes using the constructed BCKG.

To construct the $\mathrm{BCKG}$, we ingested data from a comprehensive list of structured databases: Uniprot, 
Genbank, CAZy, BRENDA, Pfam[15], PubChem[16], KEGG[17]. Furthermore, unstructured data such as dictionary of carbohydrate and scientific papers related to carbohydrates and enzymes were also ingested via CCS. Using NLP process and entity recognition, scientific data described in papers and books were extracted. Data extracted from databases, books and papers were aggregated, and links between extracted items were created on the knowledge graph using common concepts (e.g., EC number). Thus, BCKG shown in Fig. 5. was created.

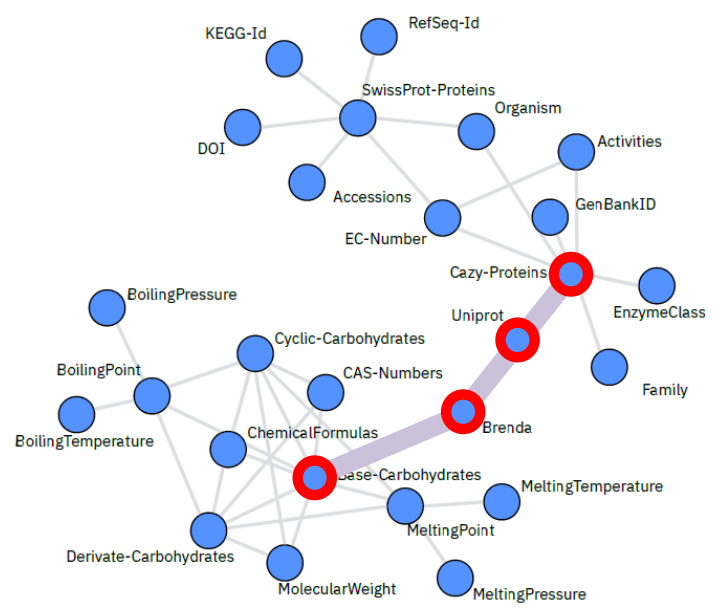

Fig. 5. An example of the BCKG.

To demonstrate the usefulness of the $\mathrm{BCKG}$, we searched for EC numbers of the enzymes synthesizing and degrading lactose which is the main cause of stomachache in adults when they drink milk. In the search using the BCKG, 6 EC numbers were obtained (Table.1). On the other hand, the search using expasy [18], which is general database for enzymatic reaction, obtained only $3 \mathrm{EC}$ numbers. The BCKG found $3 \mathrm{EC}$ numbers which could not be discovered by the search using Expasy. This result shows the usefulness and advantages of the BCKG on search by human and search using single database.

Table 1. Search results using the BCKG.

\begin{tabular}{lll}
\hline Search using the & Human search using & EC \\
BCKG & Expasy & number \\
\hline hit & No hit & 1.1 .99 .13 \\
hit & Hit & 2.4 .1 .22 \\
hit & Hit & 2.7 .1 .207 \\
hit & No hit & 3.2 .1 .23 \\
hit & Hit & 3.2 .1 .108 \\
hit & No hit & 3.2 .1 .140 \\
\hline
\end{tabular}

\subsection{Polymers KG}

Fig. 6 shows the results that CCS process the data. Subtitles, text and tables (which are regarded as labels) can be recognized. Then, the information with labels are retrieved as an output data. The approach for analyzing document structure is trained by machine learning method, so error correction enables their capability improve recognition accuracy. The recognition accuracy is over $99 \%$ in the case of applying for similar formatted documents.

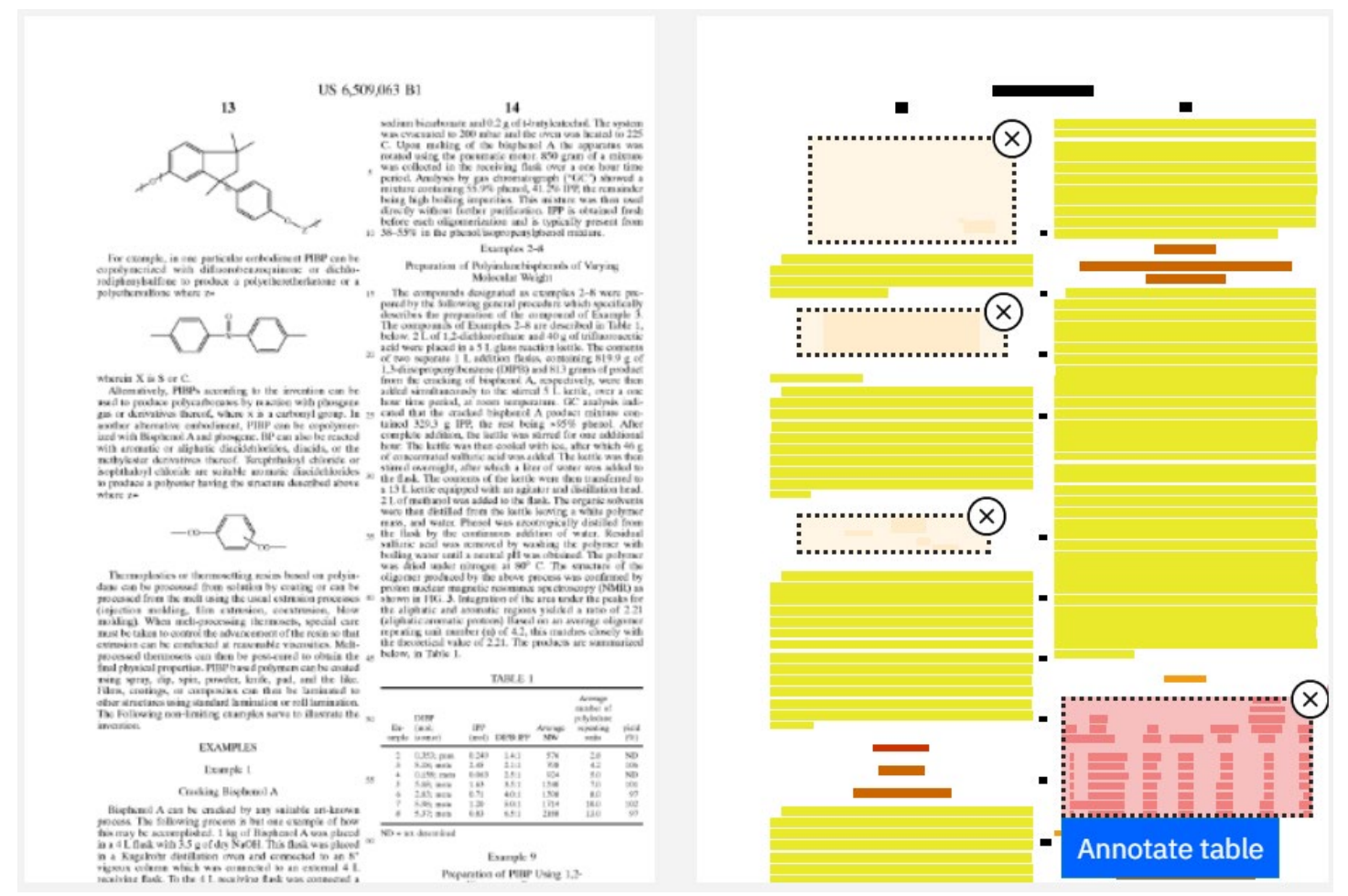

Fig. 6. Results of CCS process. 
The next step is to extract the facts that related to material development from text data. For this purpose, two technologies are needed, the commonly used natural language processing method and dictionary including technical terms (called entities) such as material names and physical property values and their relationships in the specific materials fields2) [19],[20]. In the case of text analysis, each entity is firstly extracted, the relationship among [polymer-X] to [viscosity] to $[1,000$ centistokes $]$ is then generated as shown in Fig. 7.

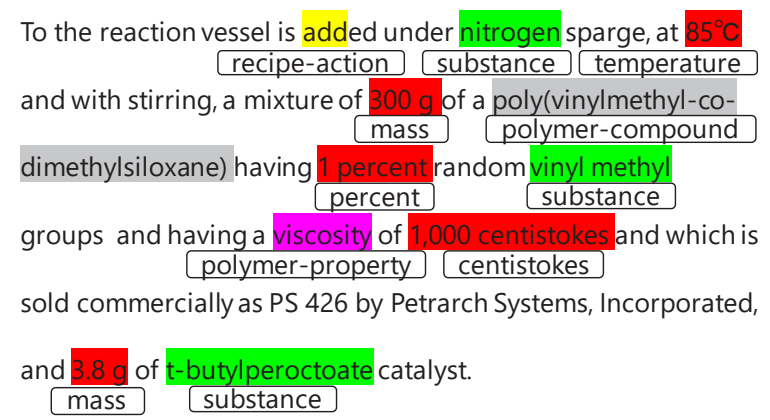

Fig. 7. Extraction of technical terms by natural language process .

Fig.8. is an example of $\mathrm{KG}$ consisting of patents related to polymers, which contains more than 400,000 data extracted from 782 patents. The entire process from ingesting patent document to build $\mathrm{KG}$ took one hour on 10 CPUs. The blue circles (nodes) are a set of entities with the same type of information, and the lines (edges) represent the relationships between entities. Paragraph node locates in the central of the $\mathrm{KG}$ and is connected to Structural information, physical properties, process conditions, and so on. Fig.9. shows the number of chemicals and their values of each property contained in $\mathrm{KG}$ on the left side. The word cloud on the right side presents the compounds associated with the glass transition temperature.

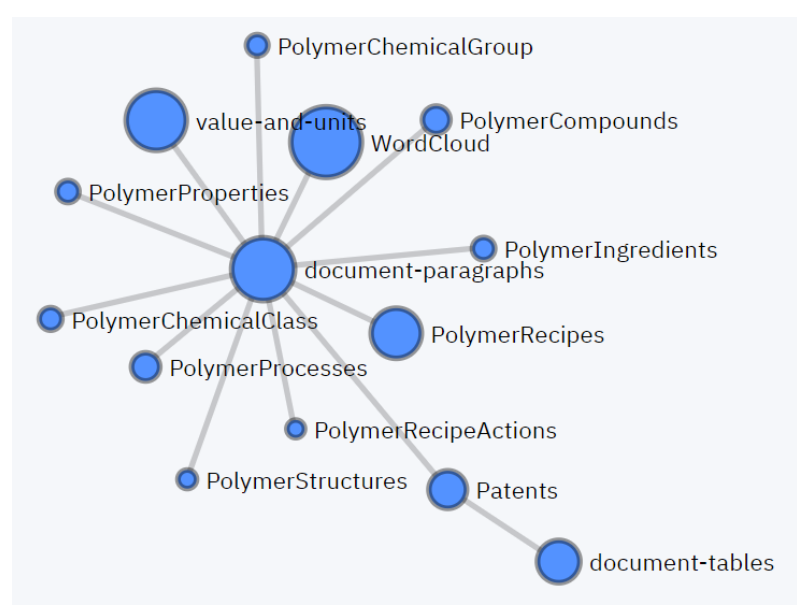

Fig. 8. KG consisting of Polymers patents.

\begin{tabular}{|c|c|c|c|}
\hline Property name & $\begin{array}{c}\# \text { of } \\
\text { chemical }\end{array}$ & $\begin{array}{l}\# \text { of } \\
\text { value }\end{array}$ & $\begin{array}{l}\text { Dolyacrylicacid } \\
\text { Cellulose Acetate } \\
\text { polyethylenesebacate }\end{array}$ \\
\hline $\begin{array}{l}\text { Glass transition } \\
\text { temperature }\end{array}$ & 49 & 2006 & $\begin{array}{c}\text { nylon66 polystyrene } \\
\text { polyscrylamide } \\
\text { polypropylene }\end{array}$ \\
\hline Thermal stability & 26 & 511 & polyvinylacetate \\
\hline Viscosity & 65 & 5954 & 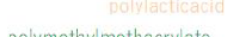 \\
\hline Tensile strength & 51 & 3076 & polybisphenolacarbonate \\
\hline
\end{tabular}

Fig. 9. statistical information stored in the KG.

\section{Overview of Platform Services for MI system}

Our Materials Informatics (MI) system is intended to be provided as SaaS (Software as a service). Since this system uses the service built on the server via the Internet, multiple authorized members can use the system in the same state. Software is regularly updated by cloud administrators and is always up to date. Since the clients' data is not stored in the terminal, the risk of data leakage can be dramatically reduced.

In Fig. 10, it shows an overview of the MI system. The MI system is managed in the cloud as a domain for each client. Each domain can only be accessed by the designated client who has been granted an account. Since the clients' private data are ingested by the clients, the private data cannot be viewed by anyone other than them, including the cloud administrator. Public data such as patents can be viewed only by the clients when the data are ingested. If there is a request from them, a service for collecting and ingesting public data will be considered in the future. 


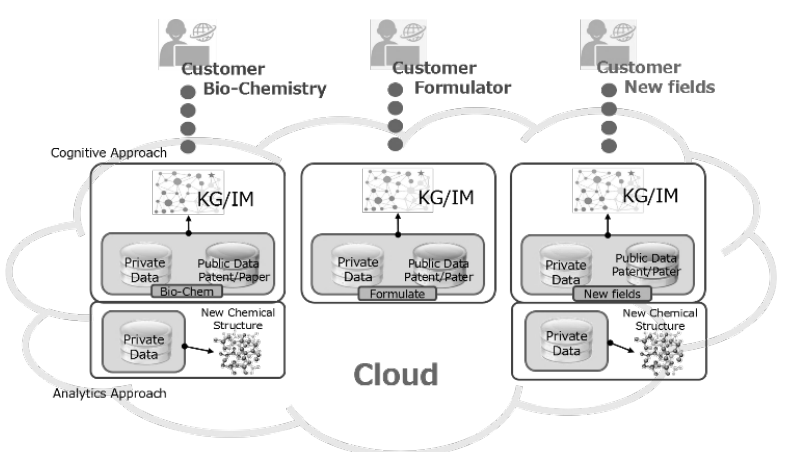

Fig. 10. Schematic image of Materials Informatics.

\section{Summary and Conclusion}

With the evolution of IoT, the advancement of $5 \mathrm{G}$, and the growth of energy consumption due to the enormous amount of data processing on servers, the increasing needs for new material development and intensifying competition in material development are inevitable. MI technology must be an indispensable technology for improving the speed of development in the search and design of such new materials, not only organic materials but also inorganic materials. And also, MI will certainly be the source of future corporate competitiveness. MI can search for target materials directly from the database which is based on conventional method depending on the intuition and experience of researchers. And it also enables efficient or direct way to develop materials to meet the needs of users. There are many advantages of MI, however we should do more for improving the systems for commonly using. For example, further improvement of data analysis technology, accumulation of data, and establishment of support system for MI introduction to users, is needed. The training of data scientists who can analyze data is also urgent. The future direction of MI evolution is the accumulation of unstructured data collected from sensing devices, combined with clustering processing by $\mathrm{AI}$, and new material simulation using quantum computers as accelerators. The field of quantum chemical calculation is also expected to be actively developed with MI field, as a more effective way to find out materials properties compare to conventional calculation methods [21].

\section{References}

1. EMC Digital Universe with Research \& Analysis by IDC, "The Digital Universe of Opportunities: Rich Data and the Increasing Value of the Internet of Things" (2014).

2. D. Reinsel, J. Grantz, and J. Rydning, "Data Age 2025, IDC (2017).

3. R. Kurzweil, "The Singularity Is Near" P.68, (2005).
4. R. Ramprasad, R. Batra, G. Pilania, A. MannodiKanakkithodi, and C. Kim, "Machine learning in materials informatics: recent applications and prospects", npj Computational Materials 3, 54 (2017).

5. A. Agrawal and A. Choudhary, "Perspective: Materials informatics and big data: Realization of the "fourth paradigm" of science in materials science", APL Materials 4, 053208 (2016).

6. C. Kirkpatrick and W. C. McMartin, Guida Chemical Space, Nature.432(7019):823-865 (2004).

7. M. Matteo, C. Auer, V. Weber, F. Zipoli, M. Dolfi, P. Staar, T. Laino, C. Bekas, A. Fujita, H. Toda, S. Hirose, and Y. Orii, An Information Extraction and Knowledge Graph Platform for Accelerating Biochemical Discoveries, Workshop on Applied Data Science for Healthcare at KDD (2019).

8. S. Takeda, T. Hama, H. H. Hsu, V. Piunova, D. Zubarev, D. Sanders, J. Pitera, M. Kogoh, T. Hongo, Y. Cheng, W. Bocanett, H. Nakashika, Y. Tsuchiya, K. Hino, K. Yano, S. Hirose, H. Toda, Y. Orii, and D. Nakano, Molecular InverseDesign Platform for Material Industries, KDD'20:Proceedings of the $26^{\text {th }} A C M$ SIGKDD International Conference on Knowledge Discovery \& Data Mining (2020).

9. P. Staar, M. Dolfi, C. Auer, and C. Bekas, "Corpus Conversion Service: A machine learning platform to ingest documents at scale", the $24^{\text {th }}$ ACM SIGKDD International Conference on Knowledge Discovery \& Data Mining (2018).

10. S. Takeda, H. Han Hsu, T. Hama, T. Yamane, K. Masuda, and D. Nakano: "New Material Search Method by Artificial Interagency", The Japan Society for Artificial Intelligence (2018).

11. A. Bateman, UniProt: a worldwide hub of protein knowledge. Nucleic Acids Research 47, D1 (2018).

12. D. A. Benson, M. Cavanaugh, K. Clark, I. Mizrachi, D.J. Lipman, J. Ostell, and E.W. Sayers, GenBank. Nucleic Acids Research 45, D1 (2017).

13. B. L. Cantarel, P. M. Coutinho, C. Rancurel, T. Bernard, V. Lombard, and B. Henrissat, The Carbohydrate-Active EnZymes database (CAZy): An expert resource for glycogenomics. Nucleic Acids Research 37, SUPPL. 1 (2009).

14.S. El-Gebali, J. Mistry, A. Bateman, S. R. Eddy, A. Luciani, S. C. Potter, M. Qureshi, L. J. Richardson, G. A. Salazar, A. Smart, E. L. Sonnhammer, L. Hirsh, L. Paladin, D. Piovesan, S. C. E. Tosatto, and R. D. Finn, A European 
ELIXIR core data resource. Nucleic Acids Research 47, D1 (2019).

15.J. Mistry, S. Chuguransky, L. Williams, M. Qureshi, G. A. Salazar, E. L. L. Sonnhammer, S. C. E. Tosatto, L. Paladin, S. Raj, L. J. Richardson, R. D. Finn, and A. Bateman, "Pfam: The protein families database in 2021", Nucleic Acids Research. 49(D1):D412-D419 (2020).

16.S. Kim, J. Chen, T. Cheng, A. Gindulyte, J. He, S. He, Q. Li, B. A. Shoemaker, P. A. Thiessen, B. $\mathrm{Yu}$, L. Zaslavsky, J. Zhang, and E. E. Bolton, "PubChem in 2021: new data content and improved web interfaces", Nucleic Acids Research. 47(D1):D1388-D1395 (2019).

17.M. Kanehisa, M. Furumichi, Y. Sato, M. Ishiguro-Watanabe, and M. Tanabe. "KEGG: integrating viruses and cellular organisms" Nucleic Acids Research. 49(D1):D545-D551 (2021).
18.P. Artinmo, M. Jonnalagedda, K. Arnold, D. Baratin, G. Csardi, E. de Castro, S. Duvaud, V. Flegel, A. Fortier, E. Gasteiger, A. Grosdidier, C. Hernandez, V. Ioannidis, D. Kuznetsov, R. Liechti, S. Moretti, K. Mostaguir, N. Redaschi, G. Rossier, I. Xenarios, and H. Stockinger, SIB bioinformatics resource portal, Nucleic Acids Res, 40(W1):W597-W603 (2012).

19. G. Lample, M. Ballesteros, S. Subramanian, K. Kawakami, and C. Dyer, Neural Architectures for Named Entity Recognition, proceedings of NAACL-HLT2016 (2016).

20. M. Honnibal and I. Montani, spaCy2: Natural language understanding with Bloom embeddings, convolutional neural networks and incremental parsing, To appear (2017).

21. H. Nishimori and M. Ozeki" Quantum computer accelerates artificial intelligence", Nikkei Business Publications, Inc. (2016) (in Japanese). 